\title{
Competing orders in thermally fluctuating superconductors in two dimensions
}

\section{Citation}

Sachdev, Subir, and Eugene Demler. 2004. "Competing Orders in Thermally Fluctuating Superconductors in Two Dimensions." Physical Review B 69 (14) (April 6). doi:10.1103/ physrevb.69.144504.

\section{Published Version}

doi:10.1103/physrevb.69.144504

\section{Permanent link}

http://nrs.harvard.edu/urn-3:HUL.InstRepos:27899432

\section{Terms of Use}

This article was downloaded from Harvard University's DASH repository, and is made available under the terms and conditions applicable to Other Posted Material, as set forth at http:// nrs.harvard.edu/urn-3:HUL.InstRepos:dash.current.terms-of-use\#LAA

\section{Share Your Story}

The Harvard community has made this article openly available.

Please share how this access benefits you. Submit a story.

\section{Accessibility}




\title{
Competing orders in thermally fluctuating superconductors in two dimensions
}

\author{
Subir Sachdev \\ Department of Physics, Yale University, P.O. Box 208120, New Haven, Connecticut 06520-8120, USA
}

Eugene Demler

Department of Physics, Harvard University, Cambridge, Massachusetts 02138, USA

(Received 6 August 2003; revised manuscript received 24 November 2003; published 6 April 2004)

\begin{abstract}
We extend recent low-temperature analyses of competing orders in the cuprate superconductors to the pseudogap regime where all orders are fluctuating. A universal continuum limit of a classical Ginzburg-Landau functional is used to characterize fluctuations of the superconducting order: this describes the crossover from Gaussian fluctuations at high temperatures to the vortex-binding physics near the onset of global phase coherence. These fluctuations induce affiliated corrections in the correlations of other orders, and in particular, in the different realizations of charge order. Implications for scanning tunneling spectroscopy and neutron-scattering experiments are noted: there may be a regime of temperatures near the onset of superconductivity where the charge order is enhanced with increasing temperatures.
\end{abstract}

DOI: $10.1103 /$ PhysRevB.69.144504

PACS number(s): 74.72.- h, 74.40.+k

\section{INTRODUCTION}

A number of recent perspectives ${ }^{1-8}$ have highlighted new experimental ${ }^{9-14}$ and theoretical ${ }^{15-27}$ works exploring the interplay between the multiple order parameters which characterize the ground state of some of the cuprate superconductors. Good evidence was obtained for a strong coupling between the superconducting order and density wave order in spin/charge/bond correlations (described more precisely below). In particular, by tuning the superconducting order by an applied magnetic field at very low temperatures $(T)$, a strong field-dependent variation was observed in the latter correlations.

In this paper, we explore the possibility of observing related connections in the finite temperature "pseudogap" region above the superconducting critical temperature $T_{c}$. Here, the superconducting order has strong $T$-dependent fluctuations; we will compute these fluctuations in the framework of a two-dimensional Ginzburg-Landau theory, including a precise characterization of strong fluctuations obtained from numerical studies. We will show that the model of Ref. 16 predicts that such fluctuations lead to a corresponding sympathetic variation in the autocorrelations of the other orders. Working to linear order in the coupling between superconductivity and these orders, we provide a computation of certain universal characteristics of the $T$ dependence of the latter fluctuations. Our results will also be formally extended to $T<T_{c}$ for completeness, but it must be noted that we neglect the interlayer coupling and quantum effects, which become important at lower $T{ }^{28}$

We begin by defining the order parameters under consideration. The primary order is the complex superconducting order $\Psi(\mathbf{r})$ which describes the spatial variation in the order associated with condensation of Cooper pairs. This is expected to undergo strong "phase" fluctuations ${ }^{29}$ for $T$ near $T_{c}$. Using the proximity of the underdoped cuprates to a superfluid-insulator quantum transition, Refs. 30,31 argued that "amplitude" fluctuations should be treated at an equal footing, ${ }^{32}$ and proposed that such thermal fluctuations could be described by a classical partition function of a suitable universal continuum limit of the Ginzburg-Landau free energy: this will be reviewed here in Sec. III. Such an approach describes the crossover from Gaussian superconducting fluctuations at temperatures well above $T_{c}$, to the vortex physics of the Kosterlitz-Thouless transition near $T_{c}$. A dynamic theory with a similar static component (although with a lattice cutoff) was recently used ${ }^{33-35}$ to describe the notable measurements ${ }^{36}$ of the Nernst effect.

This fluctuating superconductor is also expected to have appreciable correlations in other order parameters. The spindensity-wave order is described by the complex threecomponent vectors $\Phi_{x \alpha}, \Phi_{y \alpha}$, where $\alpha=x, y, z$ extends over the three spin directions, and the spin operator on site $\mathbf{r}$, $S_{\alpha}(\mathbf{r})$ is given by

$$
S_{\alpha}(\mathbf{r})=\operatorname{Re}\left[e^{i \mathbf{K}_{s x} \cdot \mathbf{r}} \Phi_{x \alpha}(\mathbf{r})+e^{i \mathbf{K}_{s y} \cdot \mathbf{r}} \Phi_{y \alpha}(\mathbf{r})\right] .
$$

Here $\mathbf{K}_{s x, y}$ are the spin-density-wave ordering wave vectors along the $x$ and $y$ principle axes of the square lattice: near a doping of $\delta=1 / 8$, we have $\mathbf{K}_{s x}=(3 \pi / 4, \pi)$ and $\mathbf{K}_{s y}$ $=(\pi, 3 \pi / 4)$. In a similar manner we can define bond order parameters $\phi_{\mathbf{a} x, y}(\mathbf{r})$ by examining the modulations in the exchange energy of a pair of spins separated by a distance $\mathbf{a}$ :

$$
S_{\alpha}(\mathbf{r}) S_{\alpha}(\mathbf{r}+\mathbf{a})=\operatorname{Re}\left[e^{i \mathbf{K}_{c x} \cdot \mathbf{r}} \phi_{\mathbf{a} x}(\mathbf{r})+e^{i \mathbf{K}_{c y} \cdot \mathbf{r}} \phi_{\mathbf{a} y}(\mathbf{r})\right] .
$$

The special case $\mathbf{a}=0$ of $\phi_{\mathbf{a x}, y}$ is a measure of the chargedensity wave order. Comparison between Eqs. (1.2) and (1.1) suggests that the ordering wave vectors are related by $\mathbf{K}_{c x, y}$ $=2 \mathbf{K}_{s x, y}$, and this is observed experimentally.

A number of other order parameters which are invariant under spin rotations, like $\phi_{\mathrm{a} x, y}$, can also be defined. ${ }^{16,21}$ These include the site charge density, the average electron kinetic energy in a bond, or modulations in the pairing amplitude. By symmetry, all such quantities will have modulations at the wave vectors $\mathbf{K}_{c x, y}$, and we can therefore expect that their order parameter fluctuations will track those of $\phi_{\mathbf{a x}, y}$. Differences in microscopic physics can, of course, 
make some of these modulations much larger than others. We will not explicitly consider all such possibilities here, and the reader should view $\phi_{\mathbf{a} x, y}$ as a suitable representative of the order parameters characterizing modulations at the wave vectors $\mathbf{K}_{c x, y}$ in observables invariant under spin rotations. We will subsequently refer to the order represented by $\phi$ simply as charge order.

While the focus of this paper is on the interplay between the superconducting fluctuations and the orders mentioned above, it should be clear to the reader that our considerations are quite general. Simple extensions lead to similar effects in the interplay of superconductivity with most of the other orders in the zoo of possibilities considered in the theory of the cuprates.

In considering correlations of $\Psi, \Phi$, and $\phi$ in the fluctuation region, it is important to consider the influence of random static impurities which are invariably present in the cuprates. As almost all impurities preserve electron number and spin rotation invariance, their influence on $\Psi$ and $\Phi$ will consist of perturbations in the random exchange class (this is discussed more explicitly in Sec. II). In contrast, the order $\phi$ breaks only lattice symmetries, and is consequently subject to the far more disruptive random field perturbations. ${ }^{37} \mathrm{In}$ two spatial dimensions, this implies that true long-range order cannot develop as $T \rightarrow 0$, and that the $\phi$ correlation length saturates at a finite value. We will assume here that there is a local onset of $\phi, \Phi$, and $\Psi$ orders at temperatures where the pseudogap develops, but at lower temperatures $\phi$ correlations are predominantly controlled by the randomfield disorder, and have only a weak, intrinsic $T$ dependence. This is also consonant with the result that thermal fluctuations are irrelevant at the random-field transition in higher dimensions ${ }^{37}$ In contrast, the fluctuations of $\Psi$ and $\Phi$ are strongly $T$ dependent, and can have an infinite correlation length as $T \rightarrow 0$. The $\Psi$ order becomes quasi-long-ranged at $T=T_{c}$ and has the strongly $T$-dependent Gaussian-to-vortex crossover noted above at $T>T_{c}$. The $\Phi$ order can also have the exponential rapid $T$ dependence associated with the breaking of $\mathrm{O}(3)$ spin rotation symmetry as $T \rightarrow 0$.

This paper will consider the regime above $T_{c}$ where

$$
\langle\Psi(\mathbf{r})\rangle=0, \quad\left\langle\Phi_{x, y \alpha}(\mathbf{r})\right\rangle=0, \quad\left\langle\phi_{\mathbf{a} x, y}(\mathbf{r})\right\rangle \neq 0 .
$$

The nonzero value $\langle\phi\rangle$ is due to the presence of randomfield perturbations which explicitly break lattice symmetries, and so allow $\phi$ to locally have a nonzero mean value which will fluctuate randomly as a function of $\mathbf{r}$. As noted above, we assume that $\langle\phi\rangle$ only has a weak intrinsic $T$ dependence. However, the fluctuations of the $\Psi, \Phi$, and $\phi$ orders are not independent, and so the strong $T$ dependence associated with the Gaussian-to-vortex crossover in $\Psi$ will induce a corresponding $T$-dependent variation in $\langle\phi\rangle$. This paper will compute this variation and suggest associated experimental tests. Strictly speaking, because there is only quasi-long-range order in $\Psi$ below $T_{c}$, the expectation values (1.3) apply also for $T<T_{c}$ : indeed, our methods and results extend also to $T<T_{c}$. However, as noted earlier, we neglect the effects of interlayer couplings and of quantum fluctuations, and so our low $T$ results should be treated with caution.
Our theory for the fluctuating orders and their interplay is summarized in Sec. II, which also contains our main results. Details of the continuum theory of the superconducting fluctuations and its Gaussian-to-vortex crossover appear in Sec. III. Section IV discusses experimental tests and possible extensions of our theory.

\section{CORRELATIONS BETWEEN FLUCTUATING ORDERS: MAIN RESULTS}

This section will introduce the free energies which control the fluctuations of the order parameters, and state our main results on the $T$ dependence of the $\phi$ order at $T>T_{c}$.

We describe the fluctuations of the superconducting order $\Psi(\mathbf{r})$ by a classical continuum partition function over the Ginzburg-Landau free energy ${ }^{30}$

$$
\begin{gathered}
\mathcal{Z}_{G L}=\int \mathcal{D} \Psi(\mathbf{r}) e^{-\mathcal{F}_{G L} /\left(k_{B} T\right)}, \\
\mathcal{F}_{G L}=\int d^{2} r\left[\frac{\hbar^{2}}{2 m^{*}}\left|\nabla_{\mathbf{r}} \Psi(\mathbf{r})\right|^{2}+a(T)|\Psi(\mathbf{r})|^{2}+\frac{b}{2}|\Psi(\mathbf{r})|^{4}\right] .
\end{gathered}
$$

We use here the notation of Refs 33-35 $m^{*}, a(T)$, and $b$ are parameters which can be computed, in principle, from the microscopic physics of the underlying electrons. The coefficient of $|\Psi(\mathbf{r})|^{2}, a(T)$, vanishes at a mean-field transition temperature, $a\left(T_{c}^{M F}\right)=0$, which will be distinct from the temperature $T_{c}$ at which there is a Kosterlitz-Thouless transition, i.e., $a\left(T_{c}\right)<0$. The purely two-dimensional, and classical theory (2.1) is expected to apply to the cuprates only for $T>T_{c}$ : below $T_{c}$ we have to also account for threedimensional effects arising from interlayer couplings, and for quantum effects at low enough $T$. All such effects will be neglected here, but for completeness, we will nevertheless discuss properties of the theory (2.1) over the full range of $T$ values.

An important point is that the functional integral in Eq. (2.1) is not defined on its own and needs an ultraviolet regulator. In the physical system this is provided by the underlying electron physics on the lattice, but this is very difficult to characterize explicitly. Here, we shall follow the procedure proposed in Ref. 30: the ultraviolet dependence can be accounted for by a suitable renormalization in the value of $a(T)$. However, because we do not know the explicit form of the ultraviolet cutoff, we cannot a priori compute the needed shift in $a(T)$. This lack of knowledge can be circumvented by using the experimental value of $T_{c}$ as an input into our calculation. The knowledge of the actual $T_{c}$, combined with the parameters in Eq. (2.1) then allows a quantitative computation of the Gaussian-to-vortex crossover with no free parameters. We reiterate that Eq. (2.1) cannot be regarded as a fully predictive theory on its own, and so cannot, even in principle, predict the actual value of $T_{c}$ : once $T_{c}$ is determined by other means, precise quantitative predictions for other observables become possible.

The Gaussian-to-vortex crossover can be expressed in terms of the following dimensionless parameter 


$$
g \equiv \frac{\hbar^{2}}{m^{*} b}\left[\frac{a(T)}{k_{B} T}-\frac{a\left(T_{c}\right)}{k_{B} T_{c}}\right] .
$$

The parameter $g$ should be a monotonically increasing function of $T$. For $T \ll T_{c}, g \sim-1 / T$, at $T=T_{c}$, we have $g=0$, and above $T_{c}, g$ takes positive values. We will see later that the present continuum theory eventually breaks down at large $T$, when $g$ begins to acquire a nonmonotonic dependence on $T$. The value of $1 /|g|$ is a measure of the strength of corrections to the mean-field theory of $\mathcal{Z}_{G L}$.

It is important to note that the $T$ dependence of $a(T)$ in Eqs. (2.1) and (2.2) is nonuniversal, and this will lead to some nonuniversality in the $T$ dependence of all our predictions. However, one of our main points is that there is a universal dependence on the parameter $g$. Moreover, once we assume a linear $T$ dependence of $a(T)$ near $T_{c}$ [as is commonly done, and we will do in Eq. (3.13)], the $T$ dependence of our predictions becomes specific.

Aided by the results of Ref. 30, 39, and 40 we will show that it is possible to obtain precise predictions for a variety of correlators of $\mathcal{Z}_{G L}$. We quote a result which will be useful in our analysis here of multiple order parameters:

$$
\frac{\hbar^{2}}{m^{*}}\left[\frac{\left\langle|\Psi|^{2}\right\rangle_{T}}{k_{B} T}-\frac{\left\langle|\Psi|^{2}\right\rangle_{T_{c}}}{k_{B} T_{c}}\right]=\mathcal{D}\left(g, T / T_{c}\right),
$$

where $\mathcal{D}\left(g, T / T_{c}\right)$ is a universal function. The averages on the left-hand side are evaluated under the partition function $\mathcal{Z}_{G L}$ at the indicated temperature. We will show in Sec. III that it is possible to reexpress the two argument function $\mathcal{D}\left(g, T / T_{c}\right)$ in terms of a single argument function $F(\mathcal{G})$ as in Eq. (3.12), where $\mathcal{G}$ depends upon $g$ and $T / T_{c}$ as in Eq. (3.11). Here we present results for the initial crossover from the Gaussian to the vortex regime, which occurs when $g$ $\gg 1$ :

$$
\begin{aligned}
\mathcal{D}\left(g, T / T_{c}\right)= & -\frac{1}{2 \pi} \ln \left(380 g T / T_{c}\right)+\frac{1}{2 \pi^{2} g} \ln \left(13.3 g T / T_{c}\right) \\
& +\frac{1}{4 \pi^{3} g^{2}}\left[\ln ^{2}\left(13.3 g T / T_{c}\right)-2 \ln \left(7.86 g T / T_{c}\right)\right] \\
& +\mathcal{O}\left(1 / g^{3}\right) .
\end{aligned}
$$

The numerical constants appearing in the arguments of the logarithms are universal. These constants, and the constants appearing in the arguments of all subsequent logarithms, depend on only two universal numbers that have to be determined by computer simulations: the latter numbers are the constant $\mathcal{G}_{c}$ computed first in Ref. 30, and the constant $\xi$ computed in Refs. 39,40. Additional higher-order terms in Eq. (2.4) have also been computed and these will be presented in Sec. III: we show there that it is possible to account for all logarithmic terms that appear at higher orders in $g$. Numerical results for the full range of values of $g$ appear in Sec. III. The expression (2.3) has ignored the possible $T$ dependencies of $m^{*}$ and $b$ for simplicity: it is possible to account for these in a similar manner, as will become clear from the discussion in Sec. III.

It is worth noting here that vortices are already present in the Gaussian theory associated with zeros of $\Psi(\mathbf{r}) .{ }^{41}$ The result (2.4) accounts for the initial correlations between these vortices, but does not include the vortex-binding physics of the Kosterlitz-Thouless transition. The latter is only accounted for by the numerical results in Sec. III.

Let us turn now to the density wave order parameters $\Phi$, $\phi$. The complete effective action for these order parameters has a rather complicated structure and was discussed in Ref. 16. A simple Gaussian form will be satisfactory for our purposes here:

$$
\begin{aligned}
\mathcal{F}_{\Phi}= & \int d^{2} r\left[K_{\Phi x}\left|\nabla_{x} \Phi_{x \alpha}\right|^{2}+K_{\Phi y}\left|\nabla_{y} \Phi_{x \alpha}\right|^{2}+\xi_{\Phi}^{-2}\left|\Phi_{x \alpha}\right|^{2}\right. \\
& \left.+h_{\Phi x}(\mathbf{r}) \Phi_{\alpha}^{2}(\mathbf{r})+h_{\Phi x}^{*}(\mathbf{r}) \Phi_{\alpha}^{* 2}(\mathbf{r})+(x \leftrightarrow y)+\cdots\right], \\
\mathcal{F}_{\phi}= & \int d^{2} r\left[K_{\phi x}\left|\nabla_{x} \phi_{\mathbf{a} x}\right|^{2}+K_{\phi y}\left|\nabla_{y} \phi_{\mathbf{a x}}\right|^{2}+\xi_{\phi}^{-2}\left|\phi_{\mathbf{a x}}\right|^{2}\right. \\
& \left.+h_{\phi x}(\mathbf{r}) \phi_{\mathbf{a} x}(\mathbf{r})+h_{\phi x}^{*}(\mathbf{r}) \phi_{\mathbf{a} x}(\mathbf{r})+(x \leftrightarrow y)+\cdots\right] .
\end{aligned}
$$

Apart from the usual Gaussian terms,${ }^{16}$ the above contains complex random fields $h_{\Phi}(\mathbf{r})$ and $h_{\phi}(\mathbf{r})$ which pin the "sliding" mode of the charge-density wave. These fields arise from impurities which preserve spin rotation invariance: as a consequence, note that the random coupling is linear in the fields $\phi$, but that there is only a random-exchange coupling to $\mathrm{O}(3)$ rotations in the spin-density wave order. These simple facts have a number of interesting implications.

(i) There can be no long-range charge order in two spatial dimensions, even at $T=0$. This implies that there can be no $T=0$ quantum critical point, tuned by the hole concentration, associated with the onset of such order. A quantum critical point associated with the restoration of $\mathrm{O}(3)$ symmetry remains possible.

(ii) The strong relevance of such random-field perturbations suggests that in the absence of couplings to other critical order parameters, the correlation length $\xi_{\phi}$ can be assumed to be roughly temperature independent at low temperatures.

(iii) The theories (2.1) and (2.5) describe a phase in which the expectation values in Eq. (1.3) hold.

Finally, as promised, let us consider the influence of the $\Psi$ fluctuations described by $\mathcal{Z}_{G L}$ on the charge order correlations. The simplest coupling between the orders is a $\lambda|\Psi|^{2}\left(\left|\phi_{\mathbf{a}}\right|^{2}+\left|\phi_{\mathbf{a} y}\right|^{2}\right)$ term, and, as in Ref. 16, this leads to the leading-order correction

$$
\xi_{\phi}^{-2}(T)=\xi_{\phi 0}^{-2}(T)+\lambda\left\langle|\Psi|^{2}\right\rangle_{T} .
$$

Here $\xi_{\phi 0}(T)$ is the "bare" correlation length of the $\phi$ order, which is expected to be only temperature dependent near $T_{c}$. We input the value of $\left\langle|\Psi|^{2}\right\rangle$ as computed in Eq. (2.3) and Sec. III, and obtain our main predictions for the superconducting fluctuation-induced modification in the $\phi$ correlation length. 


\section{CONTINUUM THEORY OF THERMAL SUPERCONDUCTING FLUCTUATIONS}

This section will review the results of Ref. 30 relevant to obtaining Eqs. (2.3) and (2.4) and its extensions. Appendix A will review the work of Prokof'ev, Ruebenacker, and Svistinov $^{39,40}$ on the dilute two-dimensional Bose gas and show that the results of their numerical simulations can be mapped onto universal quantities needed here.

Reference 30 studied the following continuum theory of a $N=2$ component real scalar $\varphi_{a}, a=1,2$ :

$$
\mathcal{F}_{\varphi}=\int d^{2} r\left[\frac{1}{2}\left(\nabla_{\mathbf{r}} \varphi_{a}\right)^{2}+\frac{\widetilde{R}}{2} \varphi_{a}^{2}+\frac{U}{24}\left(\varphi_{a}^{2}\right)^{2}\right]
$$

(Here we have changed notation for the field, from $\Phi_{\alpha}$ in Ref. 30, to $\varphi_{a}$ here, to prevent confusion with the spindensity wave order.) This theory maps onto Eq. (2.1) with the following correspondences:

$$
\begin{gathered}
\Psi=\sqrt{m^{*}}\left(\varphi_{1}+i \varphi_{2}\right) / \hbar, \\
\widetilde{R}=2 m^{*} a(T) / \hbar^{2}, \\
U=12 m^{* 2} b / \hbar^{4} .
\end{gathered}
$$

It was argued ${ }^{30}$ that the continuum limit of $\mathcal{F}_{\varphi}$ required only the single renormalization of $\widetilde{R}$ to $R$ :

$$
\widetilde{R}=R-\frac{2 k_{B} T U}{3} \int^{\Lambda} \frac{d^{2} k}{4 \pi^{2}} \frac{1}{k^{2}+R} .
$$

Here we have introduced an ultraviolet cutoff $\Lambda$ which is needed to regulate the theory $\mathcal{F}_{\varphi}$. The renormalization in Eq. (3.3) is associated with logarithmic ultraviolet divergence of the one-loop "tadpole" diagram; the renormalized $R$ in the propagator on the right-hand side accounts for tadpoles-ontadpoles, etc. All other diagrams are ultraviolet convergent and hence the simple structure of the renormalization theory.

It is important to note that (3.3) is the exact definition of $R$, and consequently $R$ is not the fully "self-energy" of the $\varphi_{a}$ field at zero external momentum; $R$ only accounts for the resummation of tadpole graphs. In practice, the relationship (3.3) implies that, when we perform a Feynman graph expansion of any observable, we can ignore all tadpole graphs, and replace $\widetilde{R}$ by $R$ in all propagators. Note also that as the bare coupling $\widetilde{R}$ extends from $-\infty$ to $\infty$, the renormalized coupling $R$ extends from 0 to $\infty$.

After the renormalization of $\widetilde{R}$ to $R$, all subsequent correlators of $\mathcal{F}_{\varphi}$ are ultraviolet convergent and so we can safely take $\Lambda \rightarrow \infty$ in them. This implies that all these correlators are universal functions of the single dimensionless quantity that can be obtained from the parameters in Eq. (3.1): this is the analog of the "Ginzburg ratio," defined here as

$$
\mathcal{G}=\frac{k_{B} T U}{R}
$$

For $T \ll T_{c}$, where $\widetilde{R} \ll 0$, we have $\mathcal{G} \rightarrow \infty$. Conversely, for $T \gg T_{c}, \widetilde{R} \gg 0$, and $\mathcal{G} \rightarrow 0$.

The field theory (3.1) exhibits a Kosterlitz-Thouless transition at some critical temperature, and the arguments above imply that this transition occurs at a universal critical value $\mathcal{G}=\mathcal{G}_{c}$. The numerical studies of Ref. 30 found $\mathcal{G}_{c} \approx 102$. The value of $\mathcal{G}_{c}$ can also be obtained from the subsequent, and more precise, numerical simulations of Refs. 39,40; this connection is discussed in Appendix A, and (A5) yields

$$
\mathcal{G}_{c}=96.9 \pm 3 \text {. }
$$

We are interested here in the value of $\left\langle\varphi_{a}^{2}\right\rangle$. This quantity requires a single additive renormalization before the continuum limit is obtained; hence we can write

$$
\frac{\left\langle\varphi_{a}^{2}\right\rangle}{k_{B} T}=2 \int^{\Lambda} \frac{d^{2} k}{4 \pi^{2}} \frac{1}{k^{2}+R}+F(\mathcal{G}),
$$

where $F(\mathcal{G})$ is a universal function. A number of analytic results for this universal function can be obtained from the methods of Ref. 30, and details appear in Appendix B. For $\mathcal{G} \rightarrow 0$ (corresponding to $T \gg T_{c}$ ), perturbation theory in powers of $U$ about the $\varphi_{a}=0$ saddle point yields

$$
F(\mathcal{G} \rightarrow 0)=\left(2.355711 \times 10^{-4}\right) \mathcal{G}^{2}+\mathcal{O}\left(\mathcal{G}^{3}\right) .
$$

All subsequent terms in the above expansion involve only integer powers of $\mathcal{G}$ and there are no logarithms. For $\mathcal{G} \rightarrow \infty$ (corresponding to $T \ll T_{c}$ ), we expand about a saddle point with $\varphi_{a} \neq 0$. As shown in Ref. 30, this is done by introducing a "dual" coupling $\mathcal{G}_{D}$ related to $\mathcal{G}$ by

$$
\frac{1}{\mathcal{G}}+\frac{1}{2 \mathcal{G}_{D}}=\frac{1}{6 \pi} \ln \left(\frac{\mathcal{G}}{\mathcal{G}_{D}}\right) .
$$

Note that as $\mathcal{G} \rightarrow \infty, \mathcal{G}_{D}=3 \pi / \ln \mathcal{G}$. For large $\mathcal{G}$, the expansion of $F$ is

$$
F(\mathcal{G} \rightarrow \infty)=\frac{1}{2 \pi} \ln \left(\frac{\mathcal{G}}{\mathcal{G}_{D}}\right)-\frac{6}{\mathcal{G}}+\mathcal{O}\left(\mathcal{G}_{D}^{2}\right) .
$$

All subsequent terms in the present expansion involve only integer powers of $\mathcal{G}_{D}$, with no additional logarithms. As discussed in Appendix A, the numerical results of Ref. 39,40 yield the values of $F$ for all values of $\mathcal{G}$. In particular, at the critical point $\mathcal{G}=\mathcal{G}_{c}$ we have from Eq. (A8)

$$
F\left(\mathcal{G}_{c}\right)=0.502 \pm 0.003 \text {. }
$$

The theory of the Kosterlitz-Thouless transition implies that $F(\mathcal{G})$ will have a weak essential singularity at $\mathcal{G}=\mathcal{G}_{c}$, similar to that in the specific heat. A plot of the values of $F(\mathcal{G})$ appears in Fig. 1. It is interesting to note that either the small $\mathcal{G}$ or the small $\mathcal{G}_{D}$ expansions is accurate for the entire range of $\mathcal{G}$ values.

The discussion so far presents our most complete results for the properties of $\mathcal{F}_{G L}$ and $\mathcal{F}_{\varphi}$ with essentially no approximations. There is, however, still some residual cutoff dependence. This can be removed by subtracting corresponding results at two different values of the bare coupling $\widetilde{R} / T$ [or 


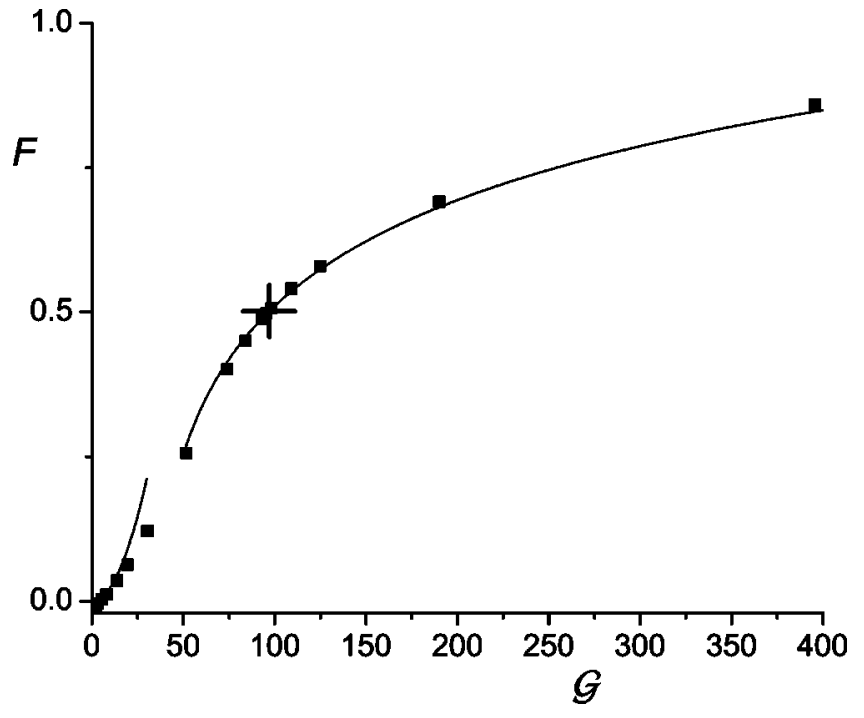

FIG. 1. Plots of the universal function $F(\mathcal{G})$. The line on the left is the small $\mathcal{G}$ approximation in Eq. (3.7). The line on the right is the large $\mathcal{G}$ approximation in Eqs. (3.8) and (3.9). The square symbols are the numerical data of Ref. 40 transformed by Eqs. (A9) and (A10). The plus marks the position of the Kosterlitz Thouless transition.

$m^{*} a(T) / T$ ], while $U$ is fixed. Depending upon the physical situation, changing $\widetilde{R} / T$ may also involve some changes in the values of $U$. However, such changes are expected to be small and we neglect any $T$ dependence in $U$ and $\Lambda$ in the remainder of this section. This allows to obtain an explicit relation between the dimensionless number $\mathcal{G}$ used in the present section, and the number $g$ in Eq. (2.2). Dividing Eq. (3.3) by $k_{B} T$ and subtracting the corresponding equation at the critical point, and using the definitions in Eqs. (3.2) and (3.4), we obtain

$$
g=\frac{6}{\mathcal{G}}-\frac{6}{\mathcal{G}_{c}}+\frac{1}{\pi} \ln \left(\frac{T \mathcal{G}_{c}}{T_{c} \mathcal{G}}\right)
$$

As expected, $g$ extends from $+\infty$ to $-\infty$ as $\mathcal{G}$ extends from 0 to $+\infty$. Applying the same procedure to Eq. (3.6) we obtain the universal function in Eq. (2.3)

$$
\mathcal{D}\left(g, T / T_{c}\right)=\frac{1}{2 \pi} \ln \left(\frac{T_{c} \mathcal{G}}{T \mathcal{G}_{c}}\right)+F(\mathcal{G})-F\left(\mathcal{G}_{c}\right)
$$

The expressions (3.5), (3.7)-(3.12) constitute the central results of this paper. Using as input the values of $g$ and $T / T_{c}$, we compute $\mathcal{G}$ from Eq. (3.11) and $\mathcal{G}_{D}$ from Eq. (3.8); then using results (3.7) and (3.9) we can compute $F(\mathcal{G})$, and finally insert in Eq. (3.12) to obtain $\mathcal{D}\left(g, T / T_{c}\right)$. In particular, the small $\mathcal{G}$ expansion in Eq. (3.7) yields Eq. (2.4). Of course, it is better to numerically solve for $\mathcal{G}$ from Eq.' (3.11), rather than obtaining the solution order-by-order in $1 / g$ as was done for Eq. (2.4).

We now present some numerical results for the parameters used by Mukerjee and Huse. ${ }^{35}$ They set $a(T)=a_{0}(T$ $-T_{c}^{M F}$ ). Inserting this in Eq. (2.2) yields

$$
g=\frac{\rho_{s}(0)}{k_{B} T_{c}}\left(1-\frac{T_{c}}{T}\right)
$$

The parametrization $a(T)=a_{0}\left(T-T_{c}^{M F}\right)$ is chosen to be valid near $T_{c}$, but can also be reasonably extended as $T \rightarrow 0$ [in BCS theory, we expect a divergent $a(T \rightarrow 0)$ $\sim-\ln (1 / T)$, but this divergence is expected to be cutoff near a superfluid-insulator transition]. By $\rho_{s}(0) \equiv-\hbar^{2} a(0) /\left(m^{*} b\right)$ in Eq. (3.13), we mean the value of the helicity modulus of $\mathcal{Z}_{G L}$ extrapolated to $T=0$ in this manner [the London penetration depth is related to the helicity modulus by $\lambda_{L}^{-2}$ $\left.=16 \pi e^{2} \rho_{s}(T) /\left(\hbar^{2} c^{2}\right)\right]$. It is worth noting here that $\rho_{s}(0)$ and $T_{c}$ are, in general, independent of each other, and the Nelson-Kosterlitz relation ${ }^{38}$ only constrains $\rho_{s}\left(T_{c}\right) / T_{c}$ $=2 / \pi$.

This framework now predicts all physical properties with two input parameters: the values of $\rho_{s}(0)$ and $T_{c}$. Mukerjee and Huse ${ }^{35}$ also defined a parameter $\eta$ as a measure of the strength of fluctuations. This is related to the parameters used here by $\eta=2 k_{B} T_{c}^{M F} / \rho_{s}(0)$. In our numerical results below, we set $\rho_{s}(0) /\left(k_{B} T_{c}\right)=(2 / \eta)\left(T_{c}^{M F} / T_{c}\right)=6.8$ following their parameters.

An important subtlety should be noted here. The use of Eq. (3.13) in Eq. (3.11) normally yields a value for $\mathcal{G}$ which decreases monotonically with increasing $T$, as seems reasonable, given our understanding of physical properties of the continuum theory. However, because the value of $g$ in Eq. (3.13) saturates as $T \rightarrow \infty$ and because of the presence of the $\ln \left(T / T_{c}\right)$ term on the right-hand side of Eq. (3.11), for very $T$ the value of $\mathcal{G}$ eventually starts increasing with increasing $T$. This is clearly unphysical and is an indication that the present continuum theory breaks down at large enough $T$. For the value of $\bar{\eta}$ being used here, this unphysical nonmonotonicity arises only at $T / T_{c}>20$, and we will therefore restrict our attention to values of $T$ below this.

Solving Eqs. (3.13) and (3.11) for $\mathcal{G}$ as a function of $T / T_{c}$, we use the results of this section and Appendix A to obtain the plot of Fig. 2 for the quantity appearing in Eq. (2.6). Note, again that either the small $\mathcal{G}$ or the large $\mathcal{G}$ expansion is reasonable accurate.

\section{CONCLUSIONS}

We conclude this paper by discussing some of the experimental and broader implications of our work. Our primary result (2.6) for the coherence length of the charge order can be tested against neutron scattering and scanning tunneling spectroscopy (STS) experiments. However, the strong random-field disorder may make $\xi_{\phi}$ inaccessible to a neutron probe which averages over the entire sample. In contrast, STS provides a local probe, and so may be more sensitive to the effects discussed here.

Consider an STS experiment with a field of view of area $A$, such as those performed in Refs. 7,13,14,42 and 43. Quasiparticle interference contributions, such as those computed in Ref. 18,44-47, appear at low temperatures, but we can expect that these will significantly broaden at temperatures above $T_{c}$. We therefore focus here only on the contribution 


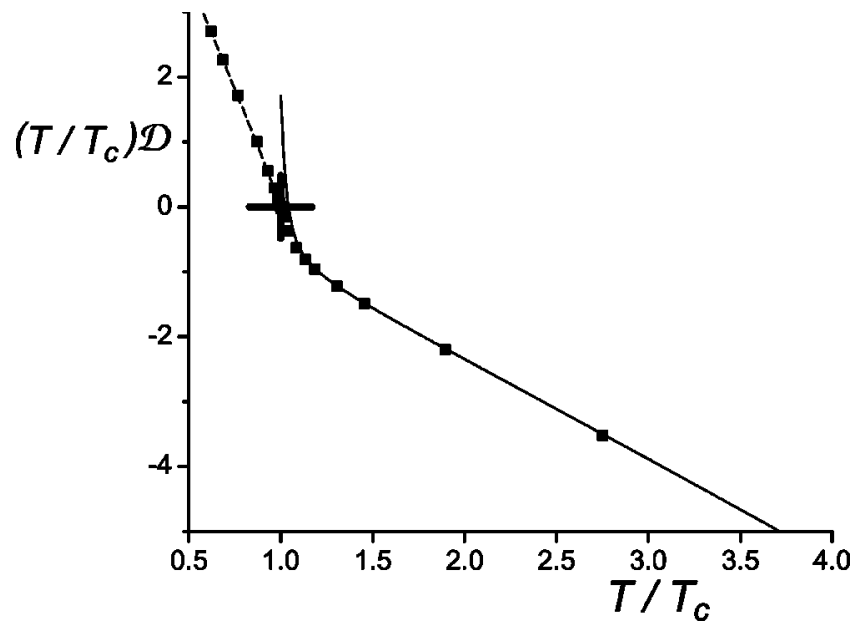

FIG. 2. Plots of the universal function $\left(T / T_{c}\right) \mathcal{D}\left(g, T / T_{c}\right)$ as a function of $T / T_{c}$ for $\rho_{s}(0) /\left(k_{B} T_{c}\right)=6.8$. From Eq. (2.3) we see that $\left\langle|\Psi|^{2}\right\rangle_{T}=\left(T / T_{c}\right)\left\langle|\Psi|^{2}\right\rangle_{T_{c}}+\left(m^{*} k_{B} T_{c} / \hbar^{2}\right)\left(T / T_{c}\right) \mathcal{D}\left(g, T / T_{c}\right)$; $\left\langle|\Psi|^{2}\right\rangle_{T}$ is determined from the above plot up to an additive, nonsingular, linear dependence on $T$ determined by $\left\langle|\Psi|^{2}\right\rangle_{T_{C}}$. This linear $T$ dependence can compensate for the linear $T$ dependence in the plot above so that $\left\langle|\Psi|^{2}\right\rangle_{T}$ saturates at high $T$. Also, as noted in the text, the present theory breaks down at large enough $T$ and its main utility is in capturing the singular increase in $\left\langle|\Psi|^{2}\right\rangle_{T}$ as $T$ crosses $T_{c}$. The solid line is the small $\mathcal{G}$ approximation obtained by solving Eqs. (3.5), (3.7), (3.10), (3.11), (3.12), and (3.13). The dashed line is the large $\mathcal{G}$ approximation obtained by solving Eqs. (3.5), (3.8), (3.9), (3.10), (3.11), (3.12), and (3.13). The square symbols are the numerical data of Ref. 40 processed via Eqs. (3.11), (3.12), (3.13), (A9), and (A10). The plus marks the position of the KosterlitzThouless transition.

of the $\phi$ fluctuations, which also lead to modulations in the local density of the states measured in STS, as shown in Ref. 18,47 . We know that the STS measurements are in the linearresponse regime. So, when we perform the Fourier transform of the local density of states at the ordering wave vector $\mathbf{K}_{c}$, we find that the signal is proportional to the uniform part of the charge order parameter, $\widetilde{\phi} \equiv \phi(\mathbf{q}=0)$. Let us estimate $\widetilde{\phi}$. We have for the free energy

$$
\langle\mathcal{F}\rangle_{\mathbf{q}=0, A} \simeq-\left\langle h^{2}\right\rangle^{1 / 2} \widetilde{\phi} A^{1 / 2}+\xi_{\phi}^{-2} \widetilde{\phi}^{2} A .
$$

Here we used that the result that the random field energy scales as the square root of the area. We can now minimize Eq. (4.1) with respect to $\widetilde{\phi}$

$$
\widetilde{\phi} \sim \frac{\left\langle h^{2}\right\rangle^{1 / 2} \xi_{\phi}^{2}}{A^{1 / 2}} .
$$

Taking $\xi_{\phi}^{2}(T)$ from Eq. (2.6) we obtain the temperature dependence of the STS signal at the wave vector $\mathbf{K}_{c}$.

For the case of competition between the superconducting $(\Psi)$ and charge $(\phi)$ orders, the coupling $\lambda$ in Eq. (2.6) will be positive. In this situation we have a seemingly counterintuitive effect: as $T$ is increased through $T_{c}$, the amplitude of the charge order is enhanced. The physical origin of this is not difficult to understand: the increase in phase coherence as
$T$ is lowered is associated with an enhanced coherent motion of the Cooper pairs, and this leads to a decrease in the amplitude of the spatial modulations. ${ }^{48}$

An alternative statement of the same physics can be made in terms of the vortices. As we argued in Ref. 16, vortices nucleate static charge order, and this was proposed as an explanation of the experiments of Ref. 13 (other approaches ${ }^{4,22,23,25-27}$ have proposed static spin order in the vortices - in our theory, static spin order is not nucleated by vortices and appears only in phases with global magnetic order $\left.{ }^{16}\right)$. Increasing $T$ above $T_{c}$ causes a proliferation of vortices and hence an enhancement of charge order.

While our discussion in this paper has been entirely at the level of the Landau theory of multiple order parameters, it is important to keep in mind that such a theory is an effective model, and does not preclude other interpretations which focus directly on the electronic quasiparticles. In particular we can view the competition between charge order and superconductivity as the competition for the ordering of lowenergy quasiparticles near the Fermi surface. So as the superconducting pairing of these quasiparticles is reduced above $T_{c}$, they are more susceptible to charge ordering.

An interesting direction for future work is to combine the continuum theory of the Ginzburg-Landau functional presented here with the theory of time-dependent superconducting fluctuations presented in Refs. 33-35: this has the prospect of placing more precise quantitative constraints on the analysis of the Nernst effect experiments. Moreover, the accuracy of either the small $\mathcal{G}$ or large $\mathcal{G}$ expansions suggests that useful analytic results may be possible. Results for the fluctuation conductivity in such an approach, including corrections to the Aslamazov-Larkin fluctuation conductivity, have appeared recently. ${ }^{49}$ A similar dynamic approach can also be applied to computing the linewidths of the electronic quasiparticles in the pseudogap regime: the strong amplitude fluctuations in $\Psi$ should lead to significant broadening in the electronic spectral functions measured in photoemission experiments.

\section{ACKNOWLEDGMENTS}

We thank A. Yazdani for numerous stimulating discussions in which he proposed a related physical picture. We also thank J. C. Davis, J. Hoffman, A. Kapitulnik, S. Kivelson, D. H. Lee, D. Podolsky, N. Prokof'ev, B. Svistinov, N.-C. Yeh, A. P. Young, and S.-C. Zhang for useful discussions. This research was supported by US NSF Grants Nos. DMR-0098226 (S.S.) and DMR-0132874 (E.D.) and the Sloan Foundation.

\section{APPENDIX A: CORRESPONDENCE WITH THE DILUTE BOSE GAS}

This appendix discusses the connection between the analysis of the dilute Bose gas in Refs. 39,40 and the results of Ref. 30 and the present paper. Let us make it clear at the outset that we are not advocating a dilute Bose gas description of the underdoped cuprates; rather, the finite temperature properties of the dilute Bose gas are characterized by some 
universal numbers which appear also in the models of interest in the present paper.

The dilute Bose gas is defined by the partition function

$$
\begin{gathered}
\mathcal{Z}_{B}=\int \mathcal{D} \psi(\mathbf{r}, \tau) e^{-\mathcal{S}_{B} / \hbar} \\
\mathcal{S}_{B}=\int_{0}^{\hbar / k_{B} T} d \tau \int d^{2} r\left[\hbar \psi^{*} \frac{\partial \psi}{\partial \tau}+\frac{\hbar^{2}}{2 m}\left|\nabla_{\mathbf{r}} \psi\right|^{2}-\mu|\psi|^{2}\right. \\
\left.+\frac{U_{B}}{2}|\psi|^{4}\right]
\end{gathered}
$$

We follow the notation of Refs. 39,40 throughout this appendix. The only exception is that the boson interaction $U$ has been replaced by $U_{B}$ to prevent confusion with the coupling $U$ in Eq. (3.1).

Integrating out the nonzero Matsubara frequency modes in the Bose gas, the action for the zero-frequency modes takes the form (3.1) with the coupling constants

$$
\begin{gathered}
-\frac{\hbar^{2} \widetilde{R}}{2 m}=\mu-2 U_{B} \int \frac{d^{2} k}{4 \pi^{2}}\left(\frac{1}{e^{\left(\hbar^{2} k^{2} /(2 m)-\mu\right) /\left(k_{B} T\right)}-1}\right. \\
\left.-\frac{k_{B} T}{\hbar^{2} k^{2} /(2 m)-\mu}\right), \\
U=\frac{12 m^{2} U_{B}}{\hbar^{4}} .
\end{gathered}
$$

The integral above is divergent in the ultraviolet, but if we use Eq. (3.3) to obtain the value of the renormalized coupling $R$ we obtain a convergent integral

$$
\begin{aligned}
R+\frac{2 m \mu}{\hbar^{2}}= & \frac{4 m U_{B}}{\hbar^{2}} \int \frac{d^{2} k}{4 \pi^{2}}\left(\frac{1}{e^{\left(\hbar^{2} k^{2} /(2 m)-\mu\right) /\left(k_{B} T\right)}-1}\right. \\
& \left.-\frac{k_{B} T}{\hbar^{2} k^{2} /(2 m)-\mu}+\frac{2 m k_{B} T / \hbar^{2}}{k^{2}+R}\right) \\
= & \frac{2 m^{2} k_{B} T U_{B}}{\pi \hbar^{4}} \ln \left(\frac{2 m \mu}{\hbar^{2} R\left(e^{\mu /\left(k_{B} T\right)}-1\right)}\right) \\
\approx & \frac{2 m^{2} k_{B} T U_{B}}{\pi \hbar^{4}} \ln \left(\frac{2 m k_{B} T}{\hbar^{2} R}\right) .
\end{aligned}
$$

In the last expression we have expanded to leading order in $U_{B}$, as required from consistency with previous approximations. Now using the definition of the dimensionless coupling $\mathcal{G}$ in Eq. (3.4), we obtain the value of the chemical potential at the Kosterlitz-Thouless transition

$$
\mu_{c}=\frac{m k_{B} T U_{B}}{\pi \hbar^{2}} \ln \left[\frac{\hbar^{2} \xi_{\mu}}{m U_{B}}\right]
$$

with universal number $\xi_{\mu}$ computed in Ref. 39 related to the universal number $\mathcal{G}_{c}$ computed earlier in Ref. 30 by

$$
\xi_{\mu}=\frac{\mathcal{G}_{c} e^{-6 \pi / \mathcal{G}_{c}}}{6} .
$$

References 39,40 obtained $\xi_{\mu}=13.3 \pm 0.4$, which is in reasonable agreement with the value $\mathcal{G}_{c} \approx 102$ obtained in Ref. 30; the latter value of $\mathcal{G}_{c}$ yields $\xi_{\mu} \approx 14.1$ from Eq. (A5).

The same method can be used to compute the boson density $n$. Integrating out the nonzero frequency modes and mapping onto the classical theory (3.1) we obtain

$$
\begin{aligned}
n= & \int \frac{d^{2} k}{4 \pi^{2}}\left(\frac{1}{e^{\left[\hbar^{2} k^{2} /(2 m)-\mu\right] / T}-1}-\frac{T}{\hbar^{2} k^{2} /(2 m)-\mu}\right)+\frac{m}{\hbar^{2}}\left\langle\varphi_{a}^{2}\right\rangle \\
= & \int \frac{d^{2} k}{4 \pi^{2}}\left(\frac{1}{e^{\left[\hbar^{2} k^{2} /(2 m)-\mu\right] / T}-1}-\frac{T}{\hbar^{2} k^{2} /(2 m)-\mu}\right. \\
& \left.+\frac{2 m k_{B} T / \hbar^{2}}{k^{2}+R}\right)+\frac{m k_{B} T}{\hbar^{2}} F(\mathcal{G}) \\
= & \frac{m k_{B} T}{2 \pi \hbar^{2}} \ln \left(\frac{2 m k_{B} T}{\hbar^{2} R}\right)+\frac{m k_{B} T}{\hbar^{2}} F(\mathcal{G}),
\end{aligned}
$$

in the last equation we have made the same simplification as that in the last equation in Eq. (A3). The result (A6) yields the expression obtained in Ref. 39 for the critical density

$$
n_{c}=\frac{m k_{B} T}{2 \pi \hbar^{2}} \ln \left(\frac{\hbar^{2} \xi}{m U_{B}}\right)
$$

with the universal number $\xi$ given by

$$
\xi=\frac{\mathcal{G}_{c}}{6} e^{2 \pi F\left(\mathcal{G}_{c}\right)} .
$$

The simulations of Refs. 39,40 obtained $\xi=380 \pm 3$, and inserting this result in Eq. (A8) allows us to compute $F\left(\mathcal{G}_{c}\right)$.

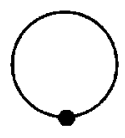

(a)

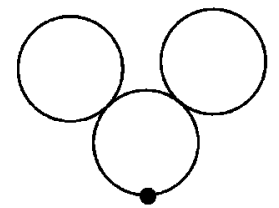

(c)

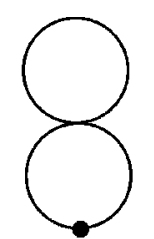

(b)

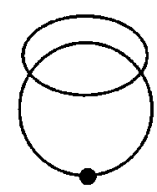

(d)

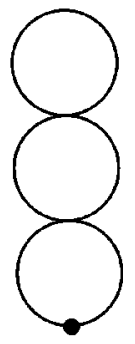

(e)
FIG. 3. Feynman graph expansion of Eq. (3.1) for the correlator (3.6). 
Finally, subtracting Eq. (A7) from the last equation in Eq. (A6) we obtain

$$
\frac{n-n_{c}}{m k_{B} T / \hbar^{2}}=\frac{1}{2 \pi} \ln \left(\mathcal{G} / \mathcal{G}_{c}\right)+F(\mathcal{G})-F\left(\mathcal{G}_{c}\right)=\lambda(X) .
$$

The function $\lambda(X)$ was computed in numerically Ref. 40, and its $\operatorname{argument} X$ can be related to our coupling $\mathcal{G}$ by (A3), yielding

$$
X=-\frac{6}{\mathcal{G}}+\frac{6}{\mathcal{G}_{c}}-\frac{1}{\pi} \ln \left(\frac{\mathcal{G}_{c}}{\mathcal{G}}\right) .
$$

These earlier results for $\lambda(X)$ therefore yield the needed function $F(\mathcal{G})$ from Eqs. (A9) and (A10).

\section{APPENDIX B: WEAK AND STRONG COUPLING EXPANSIONS}

This appendix presents discusses the expansion for the universal function $F(\mathcal{G})$ appearing in Eq. (3.6) for small and large $\mathcal{G}$.

For small $\mathcal{G}$, a simple Feynman graph expansion of Eq. (3.1) can be carried out to order $\mathcal{G}^{2}$, with the diagrams shown in Fig. 3. All propagators in Fig. 3 involve the bare "mass" $\widetilde{R}$. A simple calculation shows that the graphs $(\mathrm{a}, \mathrm{b}, \mathrm{c}, \mathrm{e})$ are all absorbed by the first term on the right-hand side of Eq. (3.6), after substitution of Eq. (3.3). This is a simple example of our claim that all "tadpole" diagrams can be neglected after substituting $R$ for $\widetilde{R}$. Only Fig. 3(d) contributes to $F(\mathcal{G})$ in Eq. (3.6) and yields

$$
\begin{aligned}
F(\mathcal{G})= & \frac{2 \mathcal{G}^{2}}{9} \int \frac{d^{2} p}{4 \pi^{2}} \int \frac{d^{2} q}{4 \pi^{2}} \int \frac{d^{2} k}{4 \pi^{2}} \\
& \times \frac{1}{\left(k^{2}+1\right)^{2}\left(q^{2}+1\right)\left[(p+k)^{2}+1\right]\left[(p+q)^{2}+1\right]} \\
= & \frac{2 \mathcal{G}^{2}}{9} \int \frac{d^{2} p}{4 \pi^{2}}\left[\frac{1}{2 \pi p \sqrt{p^{2}+4}} \ln \left(\frac{\sqrt{p^{2}+4}+p}{\sqrt{p^{2}+4}-p}\right)\right] \\
& \times\left[\frac{1}{4 \pi p\left(p^{2}+4\right)}\left\{p+\frac{2}{\sqrt{p^{2}+4}} \ln \left(\frac{\sqrt{p^{2}+4}+p}{\sqrt{p^{2}+4}-p}\right)\right\} .\right.
\end{aligned}
$$

We evaluated the last integral numerically and obtained Eq. (3.7).

For large $\mathcal{G}$, the method described in Appendix $\mathrm{C}$ of Ref. 30 was used. To one loop order, the result

$$
F(\mathcal{G})=\frac{3 R_{D}}{k_{B} T U}+\frac{1}{2 \pi} \ln \left(\frac{R}{R_{D}}\right)
$$

is easily obtained, where, as in Ref. $30, R_{D} \mathcal{G}_{D}=R \mathcal{G}$. In obtaining Eq. (B2), we have to explicitly account for all tadpole graphs, and the relationship in Eq. (2.4) of Ref. 30 between $\widetilde{R}$ and $R_{D}$. The large momentum behavior of the expansion about $\varphi_{a}=0$ and $\varphi_{a} \neq 0$ saddle points should be the same, and this ensures that the ultraviolet divergences cancel. At two loop order, 35 Feynman graphs appear; these were evaluated as in Appendix C of Ref. 30, and their sum was found to vanish. Consequently, there is no order $\mathcal{G}_{D}$ term in $F$, and the result (3.9) follows.
${ }^{1}$ J. Zaanen, Nature (London) 404, 714 (2000).

${ }^{2}$ V.J. Emery, S.A. Kivelson, and J.M. Tranquada, Proc. Natl. Acad. Sci. U.S.A. 96, 8814 (1999).

${ }^{3}$ S. Sachdev, Science 288, 475 (2000).

${ }^{4}$ S.-C. Zhang, Science 275, 1089 (1997).

${ }^{5}$ S. Sachdev and S.-C. Zhang, Science 295, 452 (2002).

${ }^{6}$ S. Sachdev, Rev. Mod. Phys. 75, 913 (2003).

${ }^{7}$ S.A. Kivelson, E. Fradkin, V. Oganesyan, I.P. Bindloss, J.M. Tranquada, A. Kapitulnik, and C. Howald, Rev. Mod. Phys. 75, 1201 (2003).

${ }^{8}$ S. Katano, M. Sato, K. Yamada, T. Suzuki, and T. Fukase, Phys. Rev. B 62, R14 677 (2000).

${ }^{9}$ B. Lake, G. Aeppli, K.N. Clausen, D.F. McMorrow, K. Lefmann, N.E. Hussey, N. Mangkorntong, M. Nohara, H. Takagi, T.E. Mason, and A. Schröder, Science 291, 1759 (2001).

${ }^{10}$ V.F. Mitrović, E.E. Sigmund, M. Eschrig, H.N. Bachman, W.P. Halperin, A.P. Reyes, P. Kuhns, and W.G. Moulton, Nature (London) 413, 501 (2001).

${ }^{11}$ B. Khaykovich, Y.S. Lee, R.W. Erwin, S.-H. Lee, S. Wakimoto, K.J. Thomas, M.A. Kastner, and R.J. Birgeneau, Phys. Rev. B 66, 014528 (2002).

${ }^{12}$ B. Lake, H.M. Rønnow, N.B. Christensen, G. Aeppli, K. Lefmann, D.F. McMorrow, P. Vorderwisch, P. Smeibidl, N.
Mangkorntong, T. Sasagawa, M. Nohara, H. Takagi, and T.E. Mason, Nature (London) 415, 299 (2002).

${ }^{13}$ J.E. Hoffman, E.W. Hudson, K.M. Lang, V. Madhavan, S.H. Pan, H. Eisaki, S. Uchida, and J.C. Davis, Science 295, 466 (2002).

${ }^{14}$ C. Howald, H. Eisaki, N. Kaneko, and A. Kapitulnik, Proc. Natl. Acad. Sci. U.S.A. 100, 9705 (2003); C. Howald, H. Eisaki, N. Kaneko, M. Greven, and A. Kapitulnik, Phys. Rev. B 67, 014533 (2003).

${ }^{15}$ K. Park and S. Sachdev, Phys. Rev. B 64, 184510 (2001).

${ }^{16}$ E. Demler, S. Sachdev, and Y. Zhang, Phys. Rev. Lett. 87, 067202 (2001); Y. Zhang, E. Demler, and S. Sachdev, Phys. Rev. B 66, 094501 (2002).

${ }^{17}$ S.A. Kivelson, D.-H. Lee, E. Fradkin, and V. Oganesyan, Phys. Rev. B 66, 144516 (2002).

${ }^{18}$ A. Polkovnikov, M. Vojta, and S. Sachdev, Phys. Rev. B 65, 220509 (2002); A. Polkovnikov, S. Sachdev, and M. Vojta, Physica C 388-389, 19 (2003).

${ }^{19}$ M. Vojta, Phys. Rev. B 66, 104505 (2002).

${ }^{20}$ J. Zaanen and Z. Nussinov, Phys. Status Solidi B 236, 332 (2003).

${ }^{21}$ D. Podolsky, E. Demler, K. Damle, and B.I. Halperin, Phys. Rev. B 67, 094514 (2003).

${ }^{22}$ Y. Chen and C.S. Ting, Phys. Rev. B 65, 180513 (2002).

${ }^{23}$ J.-X. Zhu, I. Martin, and A.R. Bishop, Phys. Rev. Lett. 89, 067003 (2002). 
${ }^{24}$ M. Franz, D.E. Sheehy, and Z. Tesanovic, Phys. Rev. Lett. 88, 257005 (2002).

${ }^{25}$ H.-D. Chen, J.-P. Hu, S. Capponi, E. Arrigoni, and S.-C. Zhang, Phys. Rev. Lett. 89, 137004 (2002).

${ }^{26}$ A. Ghosal, C. Kallin, and A.J. Berlinsky, Phys. Rev. B 66, 214502 (2002)

${ }^{27}$ B.M. Andersen, P. Hedegard, and H. Bruus, Phys. Rev. B 67, 134528 (2003).

${ }^{28}$ Three-dimensional effects are also important in a narrow window of temperatures above $T_{c}$, where the transition crosses over to the $d=3 X Y$ universality class. Such effects will be neglected here.

${ }^{29}$ V.J. Emery and S.A. Kivelson, Nature (London) 374, 434 (1995); N. Trivedi and M. Randeria, Phys. Rev. Lett. 75, 312 (1995); M. Franz and A.J. Millis, Phys. Rev. B 58, 14572 (1998); H.-J. Kwon and A.T. Dorsey, ibid. 59, 6438 (1999).

${ }^{30}$ S. Sachdev, Phys. Rev. B 59, 14054 (1999).

${ }^{31}$ S. Sachdev and O. Starykh, Nature (London) 405, 322 (2000).

${ }^{32}$ It is important to distinguish "amplitude fluctuations" in the superconducting order from the "pairing gap" of the electrons. The former measures the strength of the superconducting correlations, while the latter is an energy gap associated with the fermionic spectrum at high energies. In particular, at low $T$ near a superconductor-insulator transition it was argued in Refs. 30,31 that the amplitude fluctuations become very strong. In contrast, the gap in the electron spectrum remains finite across such a transition.

${ }^{33}$ I. Ussishkin, S.L. Sondhi, and D.A. Huse, Phys. Rev. Lett. 89, 287001 (2002).

${ }^{34}$ I. Ussishkin, Phys. Rev. B 68, 024517 (2003).

${ }^{35}$ S. Mukerjee and D.A. Huse, cond-mat/0307005 (unpublished).

${ }^{36}$ T.T.M. Palstra, B. Batlogg, L.F. Schneemeyer, and J.V. Waszczak, Phys. Rev. Lett. 64, 3090 (1990); H.C. Ri, R. Gross, F. Gollnik,
A. Beck, R.P. Huebener, P. Wagner, and H. Adrian, Phys. Rev. B 50, 3312 (1994); Z.A. Xu, N.P. Ong, Y. Wang, T. Kakeshita, and S. Uchida, Nature (London) 406, 486 (2000); Y. Wang, Z.A. Xu, T. Kakeshita, S. Uchida, S. Ono, Y. Ando, and N.P. Ong, Phys. Rev. B 64, 224519 (2001); Y. Wang, N.P. Ong, Z.A. Xu, T. Kakeshita, S. Uchida, D.A. Bonn, R. Liang, and W.N. Hardy, Phys. Rev. Lett. 88, 257003 (2002).

${ }^{37}$ Spin Glasses and Random Fields, edited by A.P. Young (World Scientific, Singapore, 1998).

${ }^{38}$ D.R. Nelson and J.M. Kosterlitz, Phys. Rev. Lett. 39, 1201 (1977).

${ }^{39}$ N. Prokof'ev, O. Ruebenacker, and B. Svistunov, Phys. Rev. Lett. 87, 270402 (2001).

${ }^{40}$ N. Prokof'ev and B. Svistunov, Phys. Rev. A 66, 043608 (2002).

${ }^{41}$ B.I. Halperin in Physics of Defects, Proceeding of the Summer School, Session 35, edited by R. Balian, M. Kleman, and J.-P. Poirier (North Holland, New York, 1981), pp. 816.

${ }^{42}$ K. McElroy, R.W. Simmonds, J.E. Hoffman, D.-H. Lee, J. Orenstein, H. Eisaki, S. Uchida, and J.C. Davis, Nature (London) 422, 592 (2003).

${ }^{43}$ M. Vershinin, S. Misra, S. Ono, Y. Abe, Y. Ando, and A. Yazdani, Science (to be published).

${ }^{44}$ Q.-H. Wang and D.-H. Lee, Phys. Rev. B 67, 020511 (2003).

${ }^{45}$ L. Capriotti, D.J. Scalapino, and R.D. Sedgewick, Phys. Rev. B 68, 014508 (2003).

${ }^{46}$ T. Pereg-Barnea and M. Franz, Phys. Rev. B 68, 180506 (2003).

${ }^{47}$ C.-T. Chen and N.-C. Yeh, Phys. Rev. B 68, 220505 (2003).

${ }^{48}$ However, it should be noted that the bare coherence length $\xi_{\phi 0}(T)$ is likely to have a $T$ dependence which acts in the opposite direction: i.e., a decrease in the amplitude of the charge order with increasing $T$. This latter effect will not be singular near $T_{c}$, but should eventually dominate at high enough $T$.

${ }^{49} \mathrm{~S}$. Sachdev, cond-mat/0308063 (unpublished). 